\title{
Prescribers' knowledge, attitudes and perceptions about blood culturing practices for adult hospitalized patients: a call for action
}

\author{
Valeria Fabre $\mathrm{MD}^{1}$, Aaron M. Milstone MD, $\mathrm{MHS}^{2}$, Sara C. Keller MD, MPH, MSPH${ }^{1}$, Karen C. Carroll $\mathrm{MD}^{3}$ and \\ Sara E. Cosgrove MD, MS ${ }^{1}$ \\ ${ }^{1}$ Division of Infectious Diseases, Department of Medicine, Johns Hopkins University School of Medicine, Baltimore, Maryland, ${ }^{2}$ Division of Infectious Diseases, \\ Department of Pediatrics, Johns Hopkins University School of Medicine, Baltimore, Maryland and ${ }^{3}$ Division of Medical Microbiology, Department of Pathology, \\ Johns Hopkins University School of Medicine, Baltimore, Maryland
}

To the Editor-The clinical variables that most commonly trigger blood cultures in daily practice (eg, fever, leukocytosis) correlate poorly with bacteremia. ${ }^{1-3}$ Published guidelines do not provide specific recommendations for when blood cultures should be drawn. ${ }^{2,4-6}$ Liberal blood culture testing may result in unnecessary and potentially harmful consequences: exposure to unnecessary antibiotics, unnecessary removal of venous catheters, overestimation of central-line-associated bloodstream infections (CLABSI) and added healthcare costs. ${ }^{7,8}$

We surveyed prescribers' knowledge, attitudes, and perceptions regarding blood cultures to help in developing future interventions aimed at optimizing blood culture testing of adult patients. Using the Qualtrics survey system, an 8-item electronic questionnaire was sent to 359 providers who provide clinical inpatient care at The Johns Hopkins Hospital: medical and surgical physician assistants and nurse practitioners (PAs/NPs), medicine house staff, hospitalists, intensivists and infectious disease (ID) physicians. We developed the survey and pilot-tested it among 7 prescribers for readability and relevance of specific items. Answers to questions that used a 5-point Likert scale were condensed into 2 categories: agree/strongly agree and neutral/disagree/strongly disagree. The survey was anonymous and voluntary. No incentives were offered for participation. Differences between groups were assessed with nonparametric tests (Fisher exact and Wilcoxon rank sum) using Stata version 13.0 software (StataCorp, College Station, TX). A 2sided $P$ value $<.05$ was considered statistically significant for all tests. The Johns Hopkins University Institutional Review Board acknowledged this study.

Overall, 109 providers (30\%) completed the survey. The median number of years of work experience for respondents was 7.5 (interquartile range [IQR], 4-12) for PAs/NPs, 5 (IQR, 3.516) for medicine attendings (hospitalists and intensivists), and 13 (IQR, 4-17.5) for ID physicians. Only 50\% of PAs/NPs responded that they would order blood cultures for a new fever in a non-ICU patient, but this proportion increased to $83 \%$ if the patient was in the ICU $(P<.01)$ (Table 1$)$. For other provider groups, $>75 \%$ of respondents would obtain blood cultures for this reason regardless of patient location. House staff and PAs/NPs were more likely to obtain blood cultures for new leukocytosis in an ICU patient than for a patient not in the ICU $(P<.01$ for house staff and

\footnotetext{
Author for correspondence: Valeria Fabre MD, Division of Infectious Diseases, Department of Medicine, Johns Hopkins University School of Medicine, 600 North Wolfe Street, Osler 425, Baltimore, Maryland 21287. E-mail: mfabre1@jhmi.edu

Cite this article: Valeria Fabre M et al. (2018). Prescribers' knowledge, attitudes and perceptions about blood culturing practices for adult hospitalized patients: a call for action. Infection Control \& Hospital Epidemiology 2018, 39, 1394-1396. doi:10.1017/ ice. 2018.224

(c) 2018 by The Society for Healthcare Epidemiology of America. All rights reserved.
}

$P=.02$ for PAs/NPs). The proportion of respondents who would order a follow-up blood culture for patients with $S$. aureus or gram-negative bacteremia in the preceding 24 hours was similar by role, regardless of ICU location. The prescribers more likely to order follow-up blood cultures for gram-negative bacteremia had less years of experience compared to the prescribers unlikely to order blood cultures for this indication (5.2 vs 9.1 years, respectively; $P<.01$ ). Single blood cultures were considered appropriate to detect bacteremia in follow-up cultures by $88 \%$ of trainees, $56 \%$ of medicine attendings, $54 \%$ of ID physicians, and $45 \%$ of PA/ NPs. More than $80 \%$ of all respondents agreed that clinicians order blood cultures reflexively in response to signs and symptoms such as fever.

Most respondents $(>85 \%)$ believed that blood cultures are expected as part of a patient's work up, and many reported that a protocol with indications would improve blood culture ordering practices ( $>80 \%$ of medicine attendings and ID physicians, $72 \%$ of PAs/NPs, and $69 \%$ of house staff). Fewer respondents felt that order sets or communication among prescribers or between prescribers and nurses would improve blood-culturing practices. Concern for missing an infection was identified by $>85 \%$ of respondents as a barrier to reducing the number of blood cultures in clinical practice. Respondents with fewer years of clinical experience agreed with this statement (7.3 years for those who agreed versus 10.5 for those who disagreed; $P=.04$ ). The consulting service was identified as a barrier to decreasing blood culture testing by $81 \%$ of attendings, $79 \%$ of PAs/NPs, $54 \%$ of ID physicians, and $51 \%$ of house staff.

Our study results suggest that decision making around blood cultures is multifactorial and is influenced by the provider's role, the provider's years of clinical experience, and patient location (ICU vs non-ICU). In this cohort, PAs/NPs had a lower tendency to order blood cultures than did providers in other roles, and ID physicians were as likely to order blood cultures than medicine attendings. Many providers thought a single set of blood cultures was adequate to detect bacteremia. This may explain trends observed in some units at our hospital, where single sets represent up to $60 \%$ of all blood cultures collected (K.C., personal communication).

In general, respondents acknowledged that febrile patients are more likely to yield positive blood cultures and the clear majority of providers indicated that they would order blood culture(s) if a patient developed a new fever. However, collecting blood during temperature spikes was not shown to increase the likelihood of documenting bacteremia. ${ }^{3}$ Clinical prediction rules to increase the positive predictive value of blood cultures have been developed; however, they have not been adopted widely in clinical practice. ${ }^{9}$ In pediatric ICU patients, a sepsis screening checklist and a clinical 
Table 1. Prescribers' Knowledge, Attitudes and Perceptions About Blood Culturing Practices for Adult Inpatients

\begin{tabular}{|c|c|c|c|c|c|}
\hline & $\begin{array}{l}\text { ID Physicians, } \\
\text { No. (\%) }\end{array}$ & $\begin{array}{l}\text { Medicine House } \\
\text { Staff, No. (\%) }\end{array}$ & $\begin{array}{l}\text { Medicine } \\
\text { Attendings, No. (\%) }\end{array}$ & $\begin{array}{l}\text { PA/NP, } \\
\text { No. }(\%)\end{array}$ & \\
\hline Questions & $(n=20)$ & $(n=35)$ & $(n=17)$ & $(n=37)$ & $P$ Value \\
\hline \multicolumn{6}{|l|}{ Likely to order blood cultures if the non-ICU patient } \\
\hline Develops new fever & $18(90)$ & $33(94)$ & $6(75)$ & $16(50)$ & $<.01$ \\
\hline Develops new hypotension & $15(75)$ & $27(77)$ & $4(53)$ & $11(31)$ & $<.01$ \\
\hline Develops new leukocytosis & $12(60)$ & $16(46)$ & $3(37)$ & $14(42)$ & .71 \\
\hline Develops persistent tachycardia & $3(15)$ & $15(43)$ & 0 & $7(21)$ & .02 \\
\hline With $S$. aureus bacteremia in the prior $24 \mathrm{~h}$ & $13(69)$ & $22(71)$ & $3(70)$ & $9(32)$ & $<.01$ \\
\hline Develops new fever & $18(90)$ & $35(100)$ & $8(80)$ & $28(83)$ & .04 \\
\hline Has persistent fever AND a negative blood culture within last $24 \mathrm{~h}$ & $13(65)$ & $23(66)$ & $4(40)$ & $17(50)$ & .24 \\
\hline Develops new hypotension & $18(90)$ & $32(91)$ & $8(80)$ & $18(51)$ & $<.01$ \\
\hline Develops new leukocytosis & $16(80)$ & $29(83)$ & $5(50)$ & $25(76)$ & .62 \\
\hline Develops persistent tachycardia & $9(45)$ & $20(57)$ & $2(20)$ & $12(36)$ & .09 \\
\hline With S. aureus bacteremia in the prior $24 \mathrm{~h}$ & $14(70)$ & $28(80)$ & $7(70)$ & $18(53)$ & .09 \\
\hline With positive blood culture with gram-negative organisms in prior $24 \mathrm{~h}$ & $12(60)$ & $28(80)$ & $6(60)$ & $16(48)$ & .03 \\
\hline All bacteremia cases need to have repeat blood cultures to document clearence & $3(15)$ & $28(80)$ & $6(37)$ & $17(46)$ & $<0.01$ \\
\hline $\begin{array}{l}\text { Clinicians order blood cultures reflexively in response to signs/symptoms such as } \\
\text { fever and hypotension }\end{array}$ & $18(90)$ & $33(94)$ & $11(69)$ & $28(76)$ & 0.23 \\
\hline \multicolumn{6}{|l|}{ Agree that the following factors influence blood culture orders: } \\
\hline Order sets & $14(74)$ & $11(31)$ & $10(63)$ & $23(62)$ & $<0.01$ \\
\hline Lack of protocol for indications for drawing blood cultures & $17(84)$ & $24(69)$ & $14(87)$ & $27(73)$ & 0.409 \\
\hline $\begin{array}{l}\text { Expectation of obtaining blood culture as part of work-up to help antibiotic } \\
\text { decisions at a later time }\end{array}$ & $17(85)$ & $33(94)$ & $13(81)$ & $31(86)$ & 0.69 \\
\hline Poor communication between physicians & $8(40)$ & $4(11)$ & $4(25)$ & $16(44)$ & 0.01 \\
\hline Poor communication between physicians and nurses & $6(23)$ & $4(11)$ & $2(12)$ & $15(43)$ & 0.02 \\
\hline Supervising physician desire for blood cultures & $13(65)$ & $20(57)$ & $5(31)$ & $28(78)$ & 0.14 \\
\hline
\end{tabular}

Note. ID, infectious disease; PA/NP, physician assistant/nurse practitioner. 
decision algorithm reduced number of blood cultures without adverse events. ${ }^{10}$ Most respondents recognized that blood cultures are ordered to help with antibiotic treatment decisions. Close monitoring of broad-spectrum antibiotic use and antibiotic deescalation should occur if interventions to limit blood culture testing are implemented.

Our study was performed at a single center and we cannot exclude volunteer bias. However, there was balanced representation from the different groups surveyed and a wide range of years of experience. In summary, more specific guidance with indications for blood cultures may help reduce unnecessary blood cultures, and interventions should include all providers, including consulting physicians.

\section{Acknowledgments.}

Financial support. Support for the statistical analysis portion of this study was provided by the National Center for Research Resources and the National Center for Advancing Translational Sciences (NCATS) of the National Institutes of Health (grant no. 1UL1TR001079). No other financial support was provided relevant to this article.

Conflicts of interest. All authors report no conflict of interest relevant to this article.

\section{References}

1. Coburn B, Morris AM, Tomlinson G, Detsky AS Does this adult patient with suspected bacteremia require blood cultures? JAMA 2012;308:502-511.
2. O'Grady NP, Barie PS, Bartlett JG, et al. Guidelines for evaluation of new fever in critically ill adult patients: 2008 update from the American College of Critical Care Medicine and the Infectious Diseases Society of America. Crit Care Med 2008;36:1330-1349.

3. Riedel S, Bourbeau P, Swartz B, et al. Timing of specimen collection for blood cultures from febrile patients with bacteremia. J Clin Microbiol 2008;46:1381-1385.

4. Miller JM, Binnicker MJ, Campbell S, et al. A guide to utilization of the microbiology laboratory for diagnosis of infectious diseases: 2018 update by the Infectious Diseases Society of America and the American Society for Microbiology. Clin Infect Dis 2018. doi: 10.1093/cid/ciy381.

5. Mermel LA, Allon M, Bouza E, et al. Clinical practice guidelines for the diagnosis and management of intravascular catheter-related infection: 2009 update by the Infectious Diseases Society of America. Clin Infect Dis 2009;49:1-45.

6. Clinical and Laboratory Standards Intitute (CLSI). Principles and procedures for blood cultures; approved guideline. Document M47-A. Wayne, PA: CLSI; 2007.

7. Alahmadi YM, Aldeyab MA, McElnay JC, et al. Clinical and economic impact of contaminated blood cultures within the hospital setting. J Hosp Infect 2011;77:233-236.

8. Zwang O, Albert RK. Analysis of strategies to improve cost effectiveness of blood cultures. J Hosp Med 2006;1:272-276.

9. Eliakim-Raz N, Bates DW, Leibovici L. Predicting bacteraemia in validated models—a systematic review. Clin Microbiol Infect 2015;21:295-301.

10. Woods-Hill CZ, Fackler J, Nelson McMillan K, et al. Association of a clinical practice guideline with blood culture use in critically ill children. JAMA Pediatr 2017;171:157-164.

\title{
An electronic antimicrobial stewardship intervention reduces inappropriate parenteral antibiotic therapy
}

\author{
Sean T. H. Liu MD, PhD, Mark J. Bailey ${ }^{1, a}$, Allen Zheng MS ${ }^{1, a}$, Patricia Saunders-Hao PharmD², \\ Adel Bassily-Marcus $\mathrm{MD}^{3}$, Maureen Harding $\mathrm{MSN}^{1}$, Meenakshi Rana $\mathrm{MD}^{1}$, Roopa Kohli-Seth $\mathrm{MD}^{3}$, Gopi Patel MD ${ }^{1}$, \\ Shirish Huprikar MD ${ }^{1}$ and Talia H. Swartz MD, $\mathrm{PhD}^{1}$ \\ ${ }^{1}$ Department of Medicine at the Icahn School of Medicine at Mount Sinai, New York, New York, ${ }^{2}$ Department of Pharmacy, Mount Sinai Hospital, New York, \\ New York and ${ }^{3}$ Department of Surgery, Icahn School of Medicine at Mount Sinai, New York, New York
}

To the Editor-A peripherally inserted central catheter (PICC) provides outpatient access to long-term parenteral antibiotics. ${ }^{1,2}$ Most antimicrobial stewardship systems do not have an outpatient parenteral antibiotic therapy (OPAT) program or mandatory infectious diseases (ID) consultation prior to patient discharge. ${ }^{3}$ Such programs ensure that PICC insertions are appropriate to avoid excessive antibiotic usage, line-associated complications, and antimicrobial resistance. ${ }^{4-6}$ Here, we report a simple intervention that identifies patients at risk for unnecessary parenteral antibiotics and recommends ID consultation.

Author for correspondence: Talia Swartz MD, Department of Medicine at the Icahn School of Medicine at Mount Sinai, New York, New York, 1 Gustave L. Levy Place, Box 1090, NY, New York 10029. E-mail: talia.swartz@mssm.edu

a Authors of equal contribution.

Cite this article: Liu STH, et al. (2018). An electronic antimicrobial stewardship intervention reduces inappropriate parenteral antibiotic therapy. Infection Control \& Hospital Epidemiology 2018, 39, 1396-1397. doi: 10.1017/ice.2018.220
The intervention resulted in an $84 \%$ relative risk reduction in patients discharged with PICC insertions without ID consultation.

The study was performed at a 1,134-bed academic medical center. Between January 2016 and May 2016, 181 PICC orders were placed for parenteral antibiotics (Fig. 1A), and 172 of these included ID consultation (95\%) while 9 (5\%) did not. Two ID attending physicians retrospectively reviewed these 9 cases and concluded that 8 PICC insertions (89\%) were inappropriate.

An electronic intervention was implemented into the PICC insertion order set in November 2016. Three prompts were inserted to ensure ID consultation for all PICC insertions for parenteral antibiotics: (1) "Will the PICC be used for IV antibiotics?" A "No" response prompted the original order set. A "Yes" prompted the following question: (2) "Is ID consulted?" A "No" prompted the statement: "ID consultation is required 